\title{
Determinação automática da velocidade instantânea para empilhamento de dados sísmicos de reflexão
}

\author{
Marcelo Santana de Souza, Michelângelo G. da Silva, e Milton J. Porsani. CPGG/UFBA, e INCT-GP/CNPq,
}

Copyright 2013, SBGf - Sociedade Brasileira de Geofísica.

Este texto foi preparado para a apresentação no VI Simpósio Brasileiro de Geofísica, Porto Alegre, 14 a 16 de outubro de 2014. Seu conteúdo foi revisado pelo Comite Técnico do VI SimBGf, mas não necessariamente representa a opinião da SBGf ou de seus associados. É proibida a reprodução total ou parcial deste material para propósitos comerciais sem prévia autorização da SBGf.

\section{Resumo}

We present a new method of automatic picking of the stacking velocities. The method consists in calculating the stacking velocities associated to the primary reflections inside a corridor (guide function) in the velocity spectra. To improve the resolution of the velocity spectra we use the logarithm of the measure of coherency, MUSIC (Multiple Signal Classification). Also to improve the signal to noise ratio of the velocity spectra, we use the Singular Value Decomposition (SVD) method, on panels of CMP corrected from normal moveout (NMO), using constant NMO velocity. The method may be applied to each CMP gather and allows to build the stacking velocity field and to obtain the zero-offset section. We applied the method in CMP gathers of the Jequitinhonha Basin to generate a velocity field and the corresponding zero-offset section. The method is both relatively simple to implement, and computationally fast.

\section{Introdução}

A obtenção de um bom campo de velocidades é um dos quesitos mais importantes no processamento sísmico CMP. Essa premissa se deve pelo fato de que a qualidade do campo de velocidades sísmicas em subsuperfície reflete diretamente na qualidade da seção zero-offset obtida, sendo importante nas etapas do empilhamento CMP e na migração. Dessa forma, a etapa da análise de velocidades torna-se imprescindível, pois quanto maior a acurácia na determinação das velocidades, ótimas para o empilhamento, melhor será o resultado da seção sísmica que se deseja obter.

Atualmente, o método mais convencional de análise de velocidades consiste em fazer o picking manual das velocidades de empilhamento nos espectros de velocidades (geralmente painéis de semblance) em algumas famílias CMPs de uma linha sísmica. Esse procedimento costuma ser trabalhoso, devido à grande quantidade de famílias CMPs.

É evidente a importância de automatizar esse processo. Neste trabalho, desenvolvemos um método de picking automático, o qual consiste em determinar automaticamente as velocidades de empilhamento, para cada amostra de tempo. Isto é feito, dentro de um corredor no espectro de velocidade que abrange à região correspondente às reflexões primárias, gerando uma função velocidade para cada família CMP analisada. Essa determinação das velocidades é feita através de uma média, ponderada pelos valores de coerência. Desta forma, as funções velocidade são determinadas automaticamente e utilizadas para compor o campo de velocidades e subsequentemente gerar a seção zero-offset.

A filtragem SVD tem sido normalmente utilizada e tem como objetivo reforçar a coerência espacial dos dados sísmicos, evidenciando as reflexões, em detrimento do ruído (Freire, 1986, Freire e Ulrych, 1988, Porsani, et al, 2010, Mojica et al, 2013, Porsani et al, 2013, Silva et al,2013). Barros, et al., 2012 e Ursin et al., 2014 utilizaram a decomposição SVD para testar diversas medidas de coerência aos painéis de semblance, junto com o power method, melhorando consideravelmente a resolução dos picos de coerência dos painéis de semblance.

Neste trabalho utilizamos o logaritmo das medidas de coerência $\left(\log _{10} M U S I C\right)$, obtidas nos painéis de velocidade constante, filtrados com o método SVD, onde apenas a primeira autoimagem é preservada (Ursin et al, 2014). Resultados sobre dados reais da Bacia do Jequitinhonha ilustram a eficácia da método proposto.

\section{O Espectro de Velocidades e a Semblance}

Podemos analisar as energias das reflexões no domínio tempo duplo de incidência normal $\left(t_{0}\right) \times$ velocidade $r m s$, gerando um espectro em que a energia de reflexão é colocada em função do tempo e da velocidade, chamado de espectro de velocidades. A geração do espectro de velocidade é feita a partir de painéis de CMP corrigidos do sobretempo normal com velocidades constante. Estes são obtidos reamostrando os traços da família CMP, ao longo de curvas hiperbólicas iniciadas no tempo $t_{0, n}$. As medidas de coerência são calculadas ao longo do tempo, para cada velocidade, desde $v_{\min }$ até $v_{\max }$, formando assim o espectro de velocidades.

As medidas de coerência são calculadas no interior de uma janela de tempo. A medida mais utilizada atualmente é a semblance como a razão normalizada entre a energia associada ao valor médio do sinal, $s_{t}$, e a energia da janela utilizada, (Neidell e Taner, 1971).

$$
S=\frac{1}{M} \frac{\sum_{t} s_{t}^{2}}{\sum_{t} \sum_{i=1}^{M} f_{i, t(i)}^{2}} .
$$

\section{Decomposição SVD Aplicada ao Painel de Semblance}

A decomposição SVD é uma operação aplicada a qualquer matriz $\mathbf{X}$, a qual expande a matriz em uma soma ponderada de matrizes de posto unitário, chamadas de autoimagens. Para uma matriz $\mathbf{X}$ de ordem $m \times n$, a decomposição SVD fatora a matriz em

$$
\mathbf{X}=\mathbf{U} \Sigma \mathbf{V}^{\mathbf{T}}
$$


sendo $\mathbf{U}$ e $\mathbf{V}$ matrizes ortogonais de ordem $m \times m$ e $n \times n$, respectivamente, logo $\mathbf{U}^{-1}=\mathbf{U}^{T}$ e $\mathbf{V}^{-1}=\mathbf{V}^{T}$. $\Sigma$ é uma matriz diagonal, retangular e do mesmo tipo de $\mathbf{X}$, cujos elementos da diagonal principal são números não negativos, chamados de valores singulares de $\mathbf{X}$.

Expressando a equação (2) como uma soma de suas autoimagens, temos:

$$
\mathbf{X}=\sum_{i=1}^{r} \sigma_{i} \mathbf{u}_{i} \mathbf{v}_{i}^{T}
$$

onde $\mathbf{u}_{i}$ é o i-ésimo autovetor de $\mathbf{X X}^{T}, \mathbf{v}_{i}$ é o i-ésimo autovetor de $\mathbf{X}^{T} \mathbf{X}$ e $\sigma_{i}$ é o i-ésimo valor singular de $\mathbf{X}$.

As primeiras autoimagens possuem uma maior coerência em relação ao sinal, enquanto que as últimas autoimagens guardam informações mais associadas ao ruído, informação incoerente.

Aplicando a decomposição SVD a cada painel de velocidade constante, a cada janela de tempo, e preservando apenas a primeira autoimagem, reforçamos a coerência do sinal e descartamos o ruído não coerente associado as demais autoimagens, aumentando assim a razão Sinal/Ruído (Ursin et al, 2014). Utilizando os painéis filtrados calculamos a seguir os valores de semblance.

\section{A Operação Logaritmo da MUSIC}

Diferente da semblance convencional, a MUSIC é definida como o inverso da projeção da janela de dados no subespaço do ruído. Matematicamente, essa medida de coerência pode ser representada pela equação,

$$
M=\frac{1}{1-S}
$$

em que $S$ é a semblance generalizada, normalizada entre 0 e 1. A MUSIC apresenta valores maiores ou iguais a 1, podendo apresentar valores muito elevados, dessa forma, uma medida plausível é aplicar o logaritmo da MUSIC, dado por:

$$
L=\log M=-\log (1-S)
$$

de forma que as regiões de maior coerência (picos) são mais realçadas em relação às regiões menos coerentes.

\section{Obtenção das Velocidades Instantâneas de Empilhamento}

O cálculo das velocidades instantâneas de empilhamento é realizado primeiramente gerando-se o corredor de velocidades, dentro do espectro de velocidades, o qual abrange a região correspondente às reflexões primárias. Dentro desse corredor, determina-se para cada amostra a velocidade instantânea de empilhamento $v_{s t}$, através do cálculo de uma média das velocidades para cada tempo $t_{i}$, dada por:

$$
\bar{v}_{s}(t i)=\frac{1}{\sum_{j=1}^{N V} S_{i j}}\left(\sum_{j=1}^{N V} S_{i j} v_{j}\right)
$$

em que $N V$ é o número de velocidades, $S_{i j}$ são os valores de coerência. Podemos entender a equação (6) como uma média das velocidades $v_{j}$, ponderada pelos valores de coerência, de modo que a média tende aos pontos mais coerentes.
Para reduzir o custo computacional, principalmente quando utilizada a Decomposição SVD, decidimos por gerar o espectro de velocidades apenas dentro do corredor no painel de semblance. As funções velocidades instantâneas de empilhamento, obtidas pelo método, são utilizadas para gerar o campo de velocidades sísmicas e corrigir as famílias CMP de NMO, empilhando-as e gerando a seção sísmica zero-offset.

\section{Resultados e Discussões}

Aplicamos o método de determinação automática das velocidades instantâneas de empilhamento em linhas sísmicas da Bacia do Jequitinhonha. A Tabela 1 apresenta os parâmetros de campo utilizados na aquisição da linha sísmica 214-298.

Tabela 1: Parâmetros de aquisição da linha sísmica 214298 da Bacia do Jequitinhonha.

\begin{tabular}{|l|c|}
\hline Número de tiros & 1004 \\
Número de canais & 120 \\
Cobertura máxima dos CMPs & 60 \\
Número de CMPs (cobertura máxima) & 1890 \\
Offset mínimo & -3720 \\
Offset máximo & -150 \\
Número de amostras & 1751 \\
Intervalo de amostragem & $4 \mathrm{~ms}$ \\
\hline
\end{tabular}

A Figura 1 mostra lado a lado uma família CMP, sua determinação automática das velocidades instantâneas e a sua posterior correção $\mathrm{NMO}$, em (a), (b) e (c), respectivamente. Observando a curva dentro do corredor em (Fig. 1b), percebemos que o cálculo da média das velocidades coincide aproximadamente com os pontos de maior coerência, correspondente às reflexões. Um fato bastante relevante para esse resultado foi a aplicação da decomposição SVD e do logaritmo da MUSIC, os quais serviram para aumentar a coerência dos painéis de semblance e realçar as regiões mais coerentes, respectivamente. Podemos perceber também que os valores de velocidade associados a um valor de coerência são tomados em cada amostra, pois a cada intervalo de amostragem, calcula-se uma nova média dentro do corredor, daí o fato das velocidades serem consideradas instantâneas. Esse é um dos quesitos que diferencia o método automático dos convencionais, já que os demais são feitos de forma manual, em que são determinados precisamente apenas alguns valores de velocidade, interpolando-se esses valores por todo espectro até gerar uma função velocidade. A Fig. 1c) ilustra a eficácia do método. Nota-se que o painel corrigido de NMO encontrase com os refletores bem horizontalizados, confirmando a boa determinação das velocidades de correção de NMO.

Para fins de comparação, utilizando o software livre de processamento Seismic Unix (SU), processamos a mesma linha sísmica em que aplicamos o método automático, realizando as etapas da análise de velocidades, correção NMO e empilhamento CMP, e por último, a geração do campo de velocidades. A análise de velocidades foi feita utilizando o programa sh-velan.sh escrito na linguagem shell script, que faz uso dos comandos do SU. Nesse programa escolhe-se um intervalo entre CMPs onde serão realizadas a análise de velocidades, então faz-se 
o picking das velocidades manualmente (obrigatoriamente em valores crescentes) dos CMPs escolhidos e interpolase as funções velocidades de modo a corresponder a toda linha sísmica. Em nosso caso, o intervalo entre CMPs escolhido foi de número 60. Para gerar o campo de velocidades, usamos o programa em shell script geracampo - bin.sh, o qual transforma as funções velocidades em um campo de velocidades em formato binário (.bin), como podemos ver na Fig. 2a). Obtidas as funções velocidades, geramos pelo Seismic Unix a seção zerooffset (Figura 3).

A Fig. 2b) mostra o campo de velocidades, obtido pela aplicação do método, considerado satisfatório pois possui valores de velocidades coerentes (valores crescentes conforme aumenta o tempo de registro), tendo como a principal vantagem 0 fato de as funções serem determinadas para todos CMPs da linha sísmica, além das velocidades serem determinadas para cada amostra. Já na Figura 4, temos a seção zero-offset da linha 214-0298, a qual apresenta uma boa continuidade dos refletores na parte de maior lâmina d'água da linha, enquanto que na parte mais rasa, o efeito do estiramento dificulta a continuidade dos refletores. Comparando com seção obtida após análise de velocidades convencional, percebemos que os métodos são equivalentes em termos de imagem, pois a depender da parte, um método imageou melhor do que o outro, em compensação, o método automático tem a vantagem de gerar um menor gasto de tempo e um menor trabalho para o intérprete.

\section{Conclusões}

O método de determinação automática das velocidades instantâneas de empilhamento, mostrou ser um método muito útil para a geração do campo de velocidades instantâneas e da seção sísmica zero-offset. O método permite diminuir consideravelmente a carga de trabalho manual da etapa de análise e determinação do campo de velocidades. Adicionalmente, o método permite a obtenção das funções velocidades para cada família CMP, e a seção empilhada, zero-offset, com baixo custo computacional.

Os resultados numéricos apresentados mostram que a seção zero-offset e o campo de velocidades obtidos com o método proposto, apresentaram resultados bastante satisfatórios. A exceção da porção superior esquerda, a seção empilhada apresenta boa continuidade dos refletores, significando que a correção $\mathrm{NMO}$, realizada com as velocidades instantâneas obtidas, de um modo geral foi boa. Também o campo de velocidades é coerente pois as velocidades estão crescentes com o aumento do tempo de registro.

O método da decomposição SVD e a aplicação do logaritmo da MUSIC foram de grande valia para a eficiência do método. $O$ método melhorou consideravelmente os valores de coerência, da mesma forma que a aplicação realçou os valores mais coerentes, de modo que se pode mapear com boa precisão os picos de coerência associados com as velocidades de empilhamento. Os resultados demonstram que o novo método é bastante promissor e estudos adicionais precisam ser realizados, tais como a melhor definição da função guia (corredor de velocidades) e a suavização do campo de velocidades obtido.

\section{Agradecimentos}

Os autores agradecem ao CPGG/UFBA/INCT-GP, pelo apoio no desenvolvimento deste trabalho. Marcelo Santana de Souza agradece ao CNPq/INCT-GP pelo suporte através da bolsa de pesquisa.

\section{Referências}

Barros, T., R. Lopes, M. Tygel, and J.T.M. Romano, (2012), Implementation aspects of eigenstructurebased velocity spectra. 74th EAGE Conference, Copenhagen, expanded abstracts.

Freire, S.L.M. (1986). Aplicações do método de decomposição em valores singulares no processamento de dados sísmicos. Tese de Doutorado, Universidade Federal da Bahia, Salvador, Brasil.

Freire, S.L.M. and T.J. Ulrych, (1988), Application of singular value decomposition to vertical seismic profiling: Geophysics, 53, 778-785.

Mojica, O.F., M.J. Porsani, e M.G. da Silva, (2013). Using SVD filters for velocity analysis and ground-roll attenuation. Revista Brasileira de Geofísica, 31, pp. 75-84.

Neidell, N.S., M.T. Taner, (1971). Semblance and other coherency measures for multichannel data, Geophysics, 36: $482-497$

Porsani, M.J., F.A.V. Artola, M. G. da Silva e P.E.M. de Melo, (2010). SVD filtering applied to improve tracking of seismic horizons. Revista Brasileira de Geofísica, 28, pp. 473-480.

Porsani, M.J., B. Ursin, M.G. Silva, and P.E.M. Melo, (2013), Dip-adaptive singular-value decomposition filtering for seismic reflection enhancement: Geophysical Prospecting, 61 no. 1, 42-52, doi: 10.1111/j.13652478.2012.01059.x

Silva, M.G. da, Porsani, M.J, (2013), Empilhamento multicmp utilizando correção NMO local e SVD: 2 Workshop da Rede Cooperativa de Pesquisa, Inovação e Transferência de Tecnologia NNE/FINEP de Geofísica de Exploração, Salvador.

Ursin, B., M. G. da Silva, M. J. Porsani, (2014). Signal and travel time parameter estimation using singular value decomposition, accept to Geophysics. 


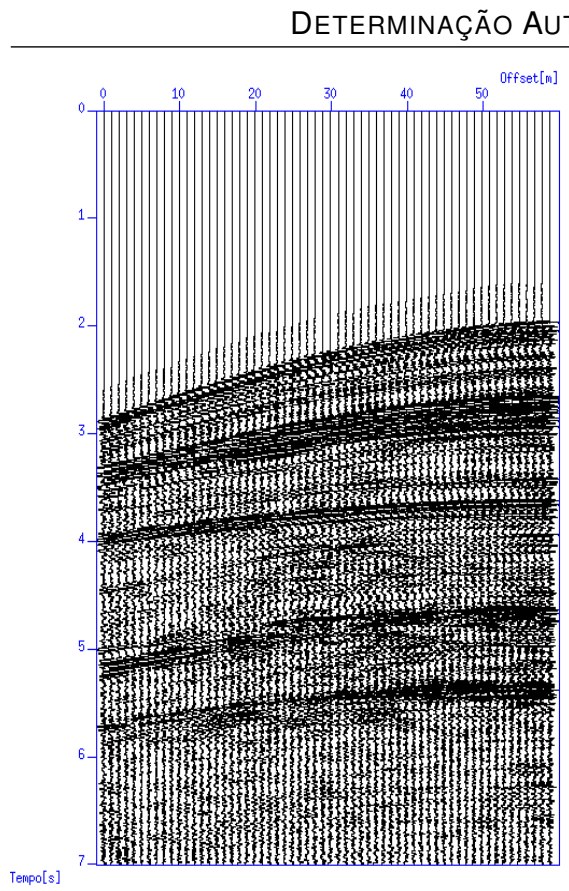

(a)

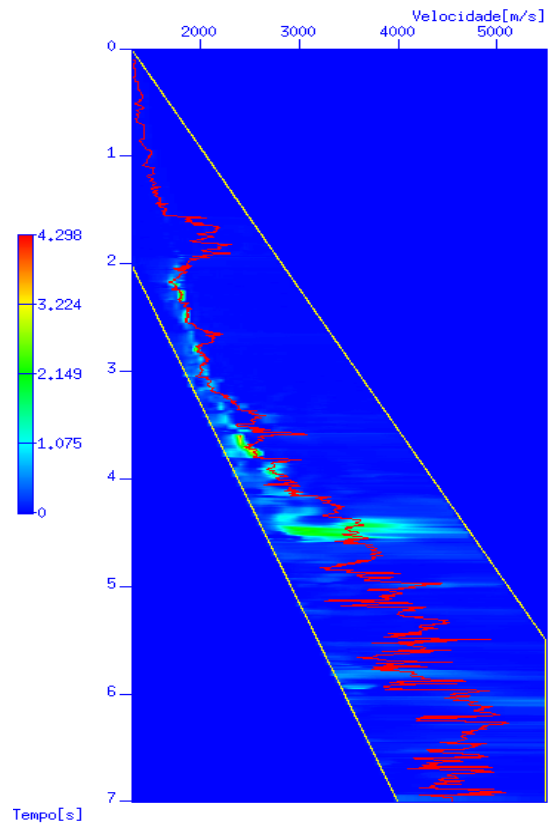

(b)

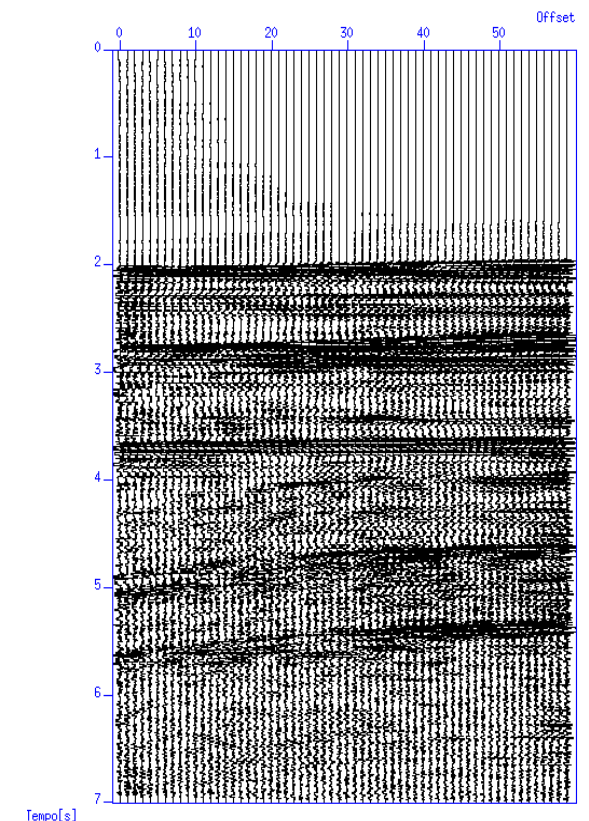

(c)

Figura 1: Família CMP em (a), a determinação das velocidades instantâneas de empilhamento em (b) e o painel corrigido de normal moveout em (c).

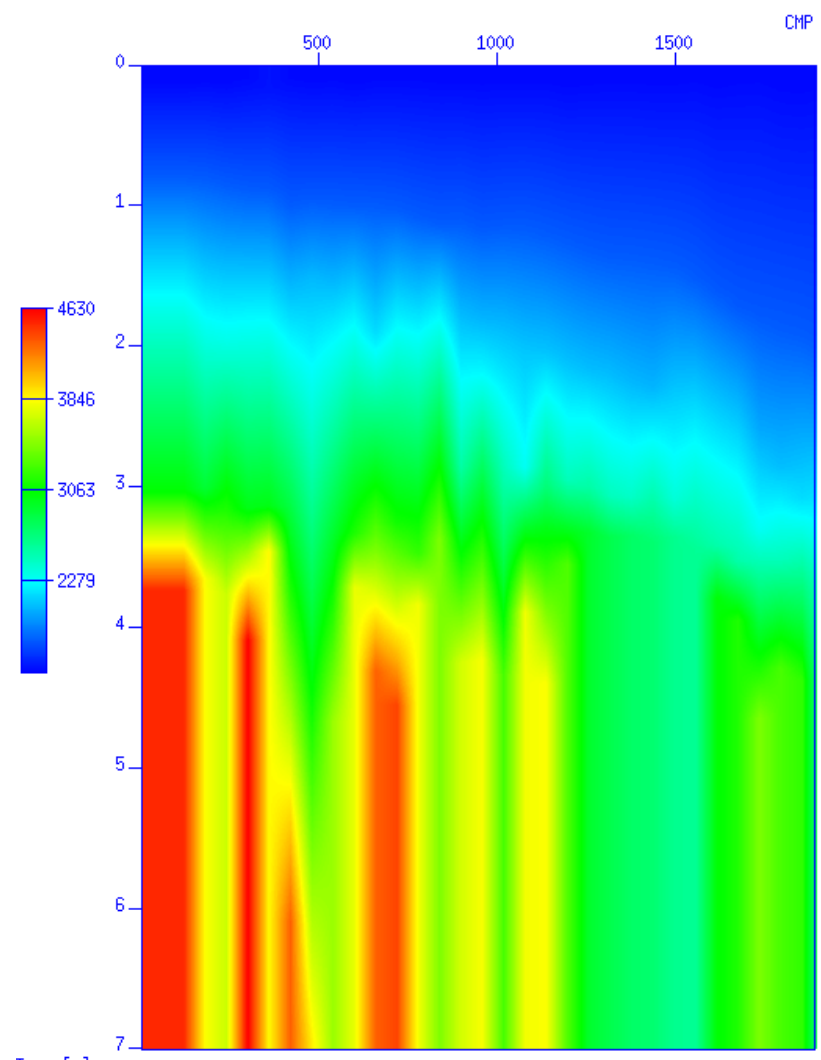

(a)

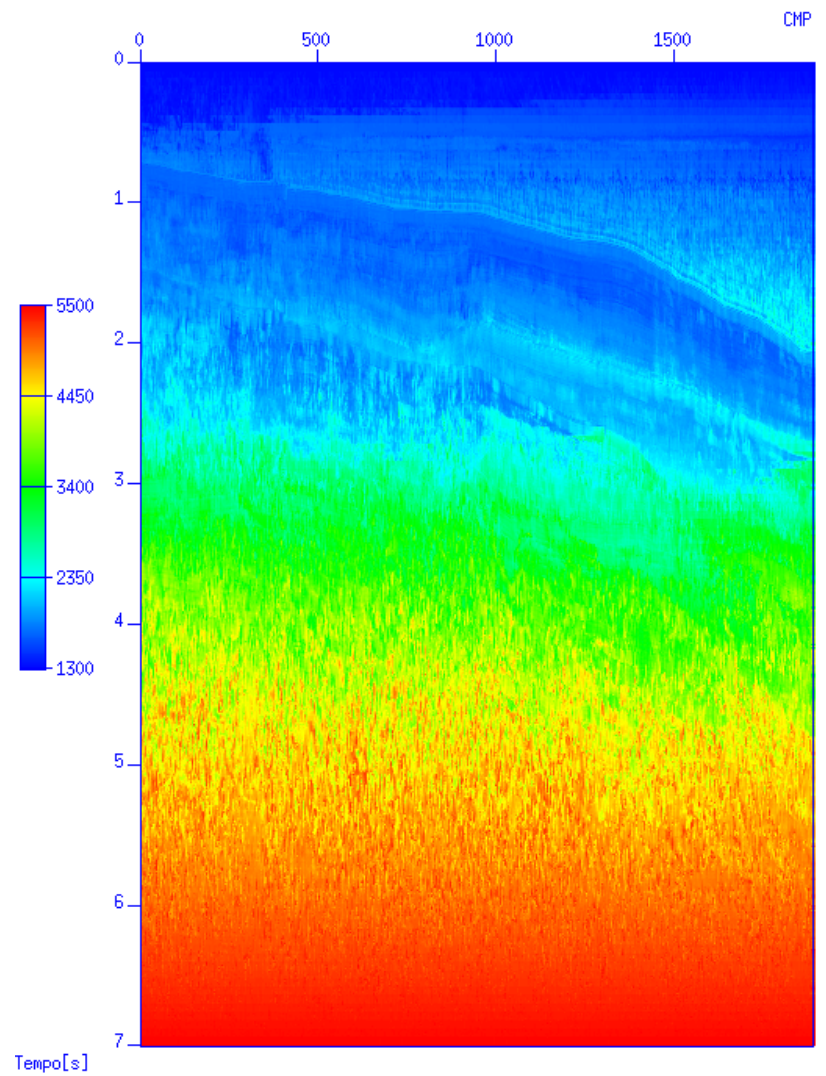

(b)

Figura 2: Campos de velocidades de empilhamento. Obtido de forma convencional através do picking manual em (a) e obtido com o método de determinação automática das velocidades instantâneas de empilhamento em (b). 


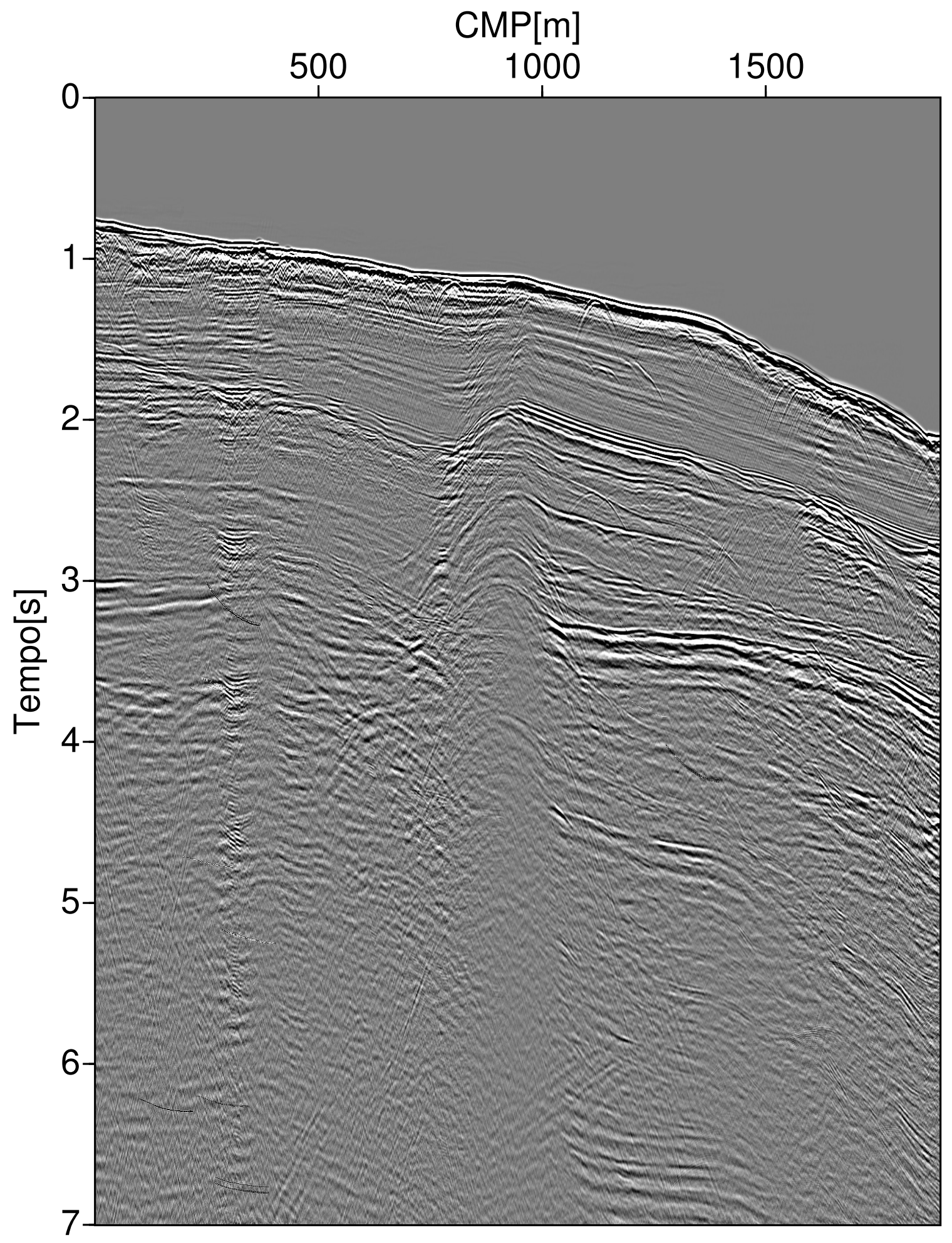

Figura 3: Seção zero-offset obtida utilizando o campo de velocidades obtido de forma convencional (Fig. 2a). 


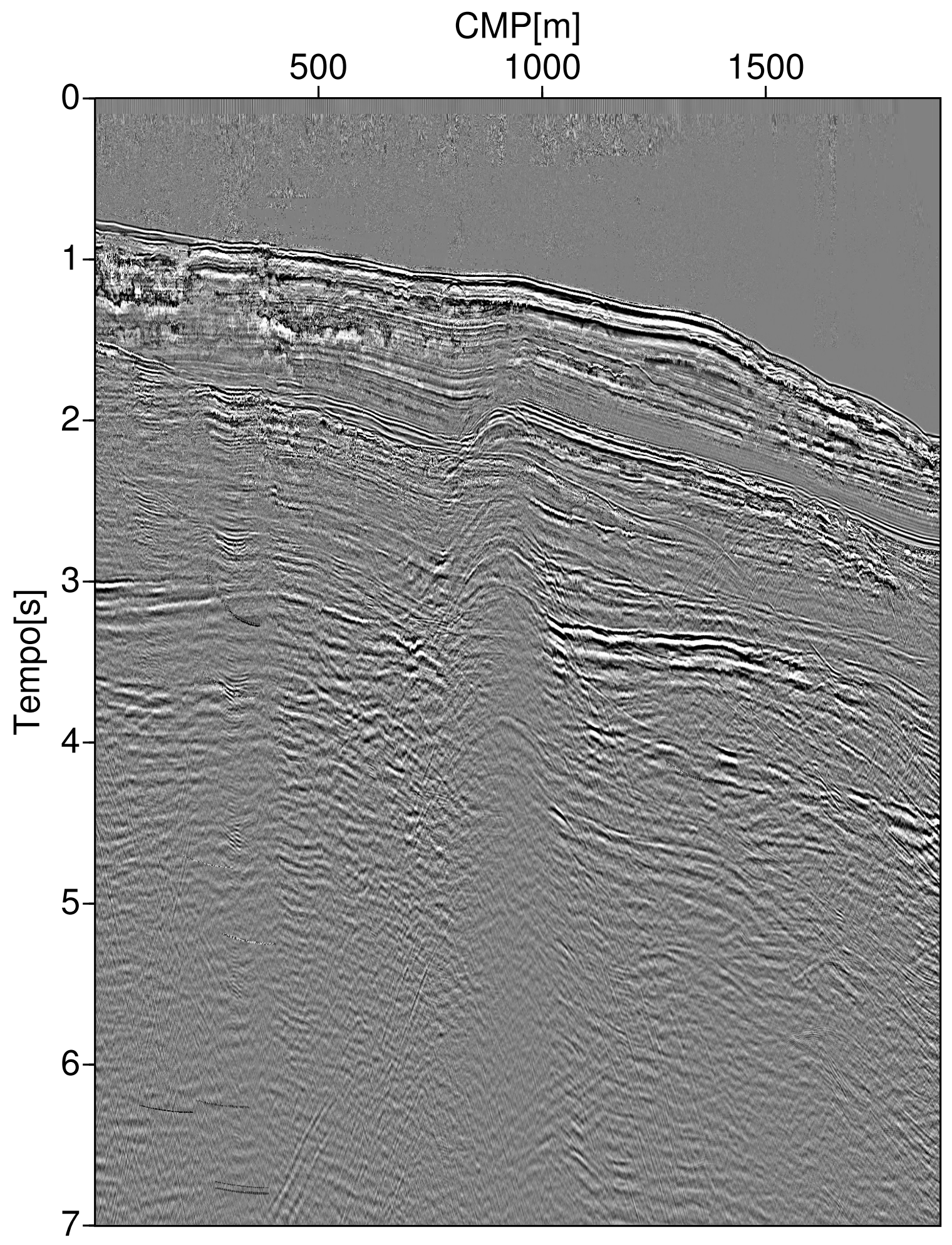

Figura 4: Seção zero-offset obtida utilizando o campo de velocidades obtido de forma automática (Fig. 2b). 